\title{
Metodologias de Aprendizagem Ativa Aplicadas à Computação: Uma Revisão da Literatura
}

\section{Active Learning Methodologies Applied to Computer Science: A Literature Review}

\author{
Avanilde Kemczinski \\ Universidade do Estado de Santa Catarina - UDESC
}

\begin{abstract}
Resumo: As metodologias ativas de aprendizagem trazem o aluno para o papel central do ensino-aprendizagem, instigando o autoaprendizado e motivando o estudante em seu próprio processo de aprendizado. $\mathrm{O}$ artigo objetiva apresentar os resultados de uma pesquisa que utilizou o método de pesquisa bibliográfica com o intuito de investigar produções acadêmicas que relataram o uso de metodologias ativas no ensino de computação, suas áreas de aplicação dentro da computação e as formas de avaliação empregadas. O levantamento bibliográfico (LB) analisou 46 produções, nas quais foi constado o emprego de metodologias ativas, identificando a PBL (Problem Based Learning) como uma das metodologias mais utilizadas. Entre as áreas de aplicabilidade na computação, o grande interesse está no ensino de programação. Isso se deve à complexidade dos conceitos e à falta de motivação dos alunos. Por fim, foi identificada a necessidade de se trabalhar com propostas de avaliações ativas e com as metodologias ativas de aprendizagem.
\end{abstract}

Palavras-chave: Metodologias Ativas, Ensino de Computação, Aprendizagem Ativa.

\begin{abstract}
The active learning methodologies brings the student to the central role of teachinglearning, instigating self-learning and motivating the student in his own teaching process. The article aims to present the results of a research that used the method of bibliographic research to investigate academic productions that reported the use of active methodologies in computer teaching, its application areas within the computation and the forms of evaluation employed. The literature review (LB) analyzed 46 productions in which the use of active methodologies was used, in which Problem Based Learning (PBL) was identified as one of the most used methodologies. Among the areas of applicability in computing the great interest is in the teaching of programming, due to the complexity of the concepts and lack of motivation of the students. Finally identified the need to work with proposals for active assessments along with active learning methodologies.
\end{abstract}

Keywords: Active Learning Methodologies, Computer Education, Active Learning. 


\section{Introdução}

As metodologias de ensino-aprendizagem vêm se adequando à realidade da sociedade atual, principalmente ao ambiente educacional na qual estão inseridas. Gil (2008) informa que a partir da segunda década do século XX, é que ocorreram grandes mudanças na didática, quando esta passa a dar valor ao princípio da atividade, ao conceito de liberdade e à individualização do aluno.

O termo aprendizagem, segundo Gil (1997), remete à aquisição de conhecimento ou de habilidades e atitudes que são resultantes de suas experiências. Bernheim e Chaui (2008) definem a aprendizagem como um processo que vem da construção do conhecimento, que é vivido e assimilado em sua estrutura cognitiva. Portanto, diante da busca do aprendizado, se faz necessária a adoção de teorias de ensino-aprendizagem para a concretização de uma educação crítica e dialética. Nessa perspectiva, compreende-se que uma metodologia de ensino deve ser adotada, buscando com isso alcançar os objetivos educacionais de aprendizagem (GIL, 1997).

Marin et al. (2010) abordam essa mudança na didática por meio da utilização de metodologias ativas, nos métodos de ensino-aprendizagem, frisando a importância do papel do aluno, e dando destaque às relações de troca de informação e comunicação. Diante dessas mudanças, o ensino da Ciência da Computação vem aplicando esse novo modelo como ferramenta para melhorar o processo de ensino-aprendizagem.

O presente artigo objetiva apresentar as metodologias ativas empregadas no ensino de Computação por meio do levantamento bibliográfico (LB), bem como observar o seu uso e a etapa de avaliação dentro do processo de ensino-aprendizagem. Esses objetivos são motivados pelo interesse de fomentar e identificar metodologias ativas para se trabalhar em sala de aula com um processo avaliativo que esteja de acordo com uma abordagem ativa.

Outros levantamentos bibliográficos referentes ao emprego de metodologias ativas podem ser encontrados em outras obras (Rocha e Lemos, 2014). Estes autores realizaram um levantamento das principais metodologias ativas aplicadas em cursos de engenharia. Fonseca e Mattar Neto (2017) apresentaram uma revisão da literatura sobre as metodologias ativas aplicadas à educação a distância, identificando as principais metodologias que se adequam à essa modalidade de ensino-aprendizagem. Já Paiva et al. (2016) fazem uma revisão das metodologias ativas, buscando identificar as modalidades de ensino-aprendizagem, os benefícios e os desafios de sua operacionalização.

Voltado à área de estudo deste artigo, é possível citar o trabalho de Silva, Pereira e Odakura (2008), que faz um mapeamento sistemático das estratégias didático-pedagógicas para o ensino-aprendizagem do pensamento computacional no Brasil, abordando também a aprendizagem ativa. Entretanto, esse trabalho é direcionado para o Ensino Fundamental. Outro estudo que se aproxima deste artigo é a publicação de Witt, Kemczinski e Santos (2018), que apresenta o emprego de metodologias de resolução de problemas para o ensino de computação focado na PBL (Problem Based Learning).

As metodologias ativas no ensino de Computação têm sido um tema de estudo que se ampliou nos últimos anos. Observa-se com isso o interesse de pesquisadores na busca de 
alternativas para os problemas clássicos da área, como a evasão e a falta de motivação dos estudantes. Assim, o presente artigo faz um levantamento bibliográfico para identificar as metodologias ativas que dão suporte ao processo de ensino-aprendizagem da área de computação em diversos níveis de ensino. Para tanto, o artigo está dividido em: fundamentação, na seção 2; procedimentos metodológicos, na seção 3; resultados, na seção 4; e, por fim, a conclusão, na seção 5, seguida das referências bibliográficas.

\section{Fundamentação}

O avanço no processo de ensino-aprendizagem é uma atividade que exige esforço (Sobral e Campos, 2012). Há a proposta de romper com o modelo tradicional de ensino, que visa transmitir o conhecimento por meio da fala do professor para o aluno. A aprendizagem ativa vem com a ideia de mudar o papel central no processo de ensino e aprendizagem, colocando o aluno como protagonista. Gil (2008) menciona que foi a partir da segunda década do século XX que se desenvolveu esse movimento ou essa mudança, partindo para as metodologias ativas.

A mudança veio com a necessidade de "envolver o aluno enquanto protagonista da sua aprendizagem, desenvolvendo ainda o senso crítico diante do que é aprendido, bem como competências para relacionar esses conhecimentos ao mundo real" (PINTO et al., 2012, p.78). Portanto, o professor passa a ser visto como um mediador, com a responsabilidade de preparar e orientar as pessoas para a vida em sociedade, deixando de ser o elemento central e passando a ser um facilitador da aprendizagem. Bellan (2008, p. 56) destaca que "o papel do facilitador é apresentar informações por meio de técnicas de ensino e criar um ambiente adequado para a aprendizagem".

Assim, a aprendizagem ativa, conforme apontam Barbosa e Moura (2013), ocorre no momento em que o aluno interage com o assunto em estudo, seja ouvindo, falando, perguntando, discutindo, fazendo ou ensinando. O estudante é estimulado a construir o seu próprio conhecimento e não somente recebê-lo de forma passiva pelo professor. Na explicação, os autores supracitados reforçam que nesse ambiente de aprendizagem ativa o papel do professor muda para o papel de orientador, supervisor, facilitador do processo de aprendizagem, tirando-o da função de ser apenas fonte única de informação e conhecimento. Sendo assim, a aprendizagem ativa é obtida por meio do uso de metodologias ativas no processo de ensino-aprendizagem.

Mitre et al. (2008) propõem, em sua perspectiva de educação, que o aluno tem que possuir a capacidade de autogerenciar o seu processo de formação. Para que isso ocorra, deve ser inserido no processo de forma ativa. De acordo com Berbel (2011), isso pode ocorrer por meio da utilização de experiências reais ou simuladas, com a resolução de desafios encontrados no ambiente social do aluno e nos mais diferentes contextos.

Diante disso, Kane (2004) define características importantes, a exemplo de: (a) procurar incentivar o pensamento independente e crítico; (b) encorajar os estudantes a assumirem a responsabilidade por seu aprendizado; (c) inserir os estudantes em atividades, garantindo a eles o papel de protagonista; (d) considerar importante o papel do educador como promotor e organizador das atividades. 
A metodologia ativa vem com um caráter motivacional para o aluno. Atualmente, o curso de computação no Brasil tem obtido índices altos de reprovação, o que afeta o processo de aprendizado dos alunos. Ramos et al. (2015) apresentam uma revisão sistemática de âmbito nacional, que aponta uma média de $45,6 \%$ de estudantes reprovados quando se trata da abordagem de estudo tradicional. Destacam, porém, uma diminuição para 32,6\% nos índices de reprovação quando são empregadas estratégias diferenciadas.

Moran (2015) afirma que "alguns componentes são fundamentais para o sucesso da aprendizagem: a criação de desafios, atividades, jogos que realmente trazem as competências necessárias para cada etapa". Essas propostas ativas acabam se tornando mais eficazes que as tradicionais (Ramos et al., 2015). Para se alcançar a aprendizagem ativa, existem muitas teorias e métodos que podem ser trabalhados. Uma representação dessas relações pode ser vista na diagramação de Bishop e Verleger (2013), conforme a Figura 1.



Fonte: Bishop e Verleger (2013, p. 7).

O esquema (Figura 1) de Bishop e Verleger (2013) apresenta a complexidade da relação das teorias de aprendizagem, e como elas estão inseridas no contexto das metodologias ativas de aprendizagem. Na Figura 1 pode-se observar a intersecção entre as metodologias de Problem Based Learning e Colaborative Learning, pois ambas possuem características semelhantes na sua definição, a exemplo da utilização de grupos de trabalho.

Grabinger e Dunlap (1995) apontam que as metodologias ativas são apoiadas pela Teoria de Aprendizagem Construtivista. Piaget (2011) explica que o aprendiz, nessa teoria, passa a ter um papel ativo em seu aprendizado, no qual ele constrói o seu conhecimento. Conforme Mizukami (1986), o aluno ativo exerce as funções de observar, experimentar, comparar, relacionar, analisar, justapor, compor, levantar hipóteses e argumentar, sendo ativo no seu processo de aprendizagem.

Entre as metodologias ativas (MA), podemos elencar algumas, tais como (do inglês): Problem-Based Learning (PBL), Peer-Assisted Learning (PAL), Cooperative Learning e Collaborative Learning (CL) e Peer Tutoring (PT). Sabendo da existência dessas práticas ativas de ensino-aprendizagem, na seção seguinte apresentaremos os procedimentos metodológicos, a fim de investigar essas propostas empregadas no ensino de Computação.

\section{Procedimentos Metodológicos Adotados}


Para conduzir a pesquisa, foi necessário definir os métodos e condições que permitiriam identificar materiais que agreguem informações úteis ao objetivo central de estudo. O processo adotado para isso pode ser observado na Figura 2.

Figura 2 - Procedimentos Metodológicos

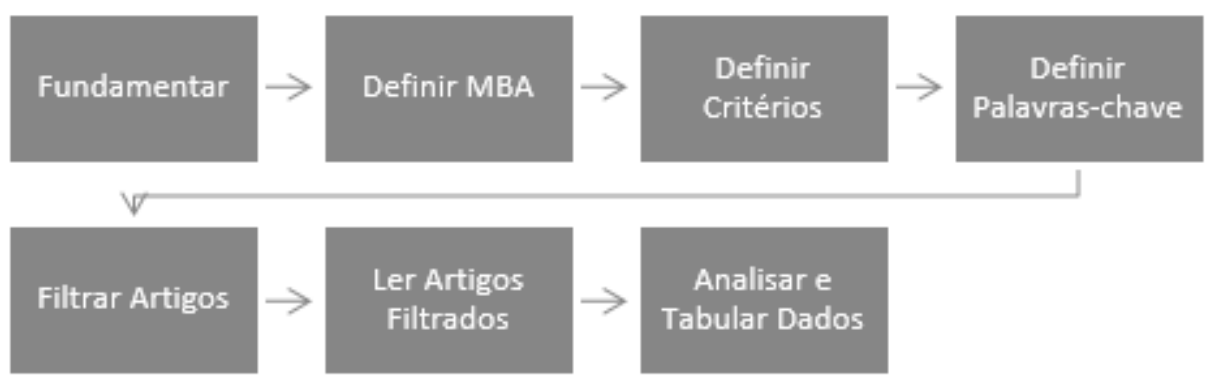

Fonte: os autores.

O primeiro passo adotado foi a fundamentação sobre a definição de metodologias ativas de aprendizagem. Após o estudo sobre as metodologias ativas, foram definidos os mecanismos de busca acadêmica (MBA) a serem utilizados. Os mecanismos para esta pesquisa foram: Scholar, ACM, Periódicos CAPES e ScienceDirect. De acordo com Buchinger et al. (2014), esses mecanismos estão entre os 10 melhores e mais importantes em termos de busca por conteúdo científico, considerando recursos de busca, de refinamento e auxiliares.

Para que fossem selecionados os materiais condizentes com a pesquisa, definiu-se como palavras-chave de busca os termos "Ciência da Computação", "Ensino de Computação", "Aprendizagem Ativa" e "Metodologia Ativa". A escolha das palavras-chave ocorreu por serem a forma mais genérica dos temas abordados e que também traziam um maior número de resultados na filtragem das buscas.

Para a investigação bibliográfica tradicional foram definidos critérios para identificar se o material da base de dados é considerado válido. Assim, os artigos selecionados foram publicações em inglês, português e espanhol, publicadas após 2007, disponíveis para download e com mais de 4 páginas. Para a seleção dos trabalhos, os autores fizeram uma leitura do título, do resumo e da metodologia de cada trabalho, e escolheram aqueles que apresentavam metodologias ativas empregadas no ensino de computação (sem restrição quanto ao nível de ensino) e que contivessem a descrição do processo metodológico utilizado.

Após a filtragem inicial, foram lidos na íntegra 46 artigos com informações consideradas relevantes à pesquisa (https://goo.gl/rJ4eoA) - A partir dessas leituras, os dados foram extraídos e tabulados para serem analisados, conforme pode ser visto na seção 4 .

\section{Resultados}

Esta seção apresenta os resultados observados com base nos dados coletados. A pesquisa foi executada no primeiro semestre de 2018. O primeiro item observado nas produções selecionadas foi que propostas de ensino-aprendizagem ativas estão sendo empregadas no 
ensino de computação. Com base nos artigos, constatou-se que a técnica mais empregada foi a PBL (Problem Based Learning), com 9 publicações, conforme pode ser visto na Figura 3.

Figura 3 - Proposta de Ensino-Aprendizagem Ativas Mapeadas

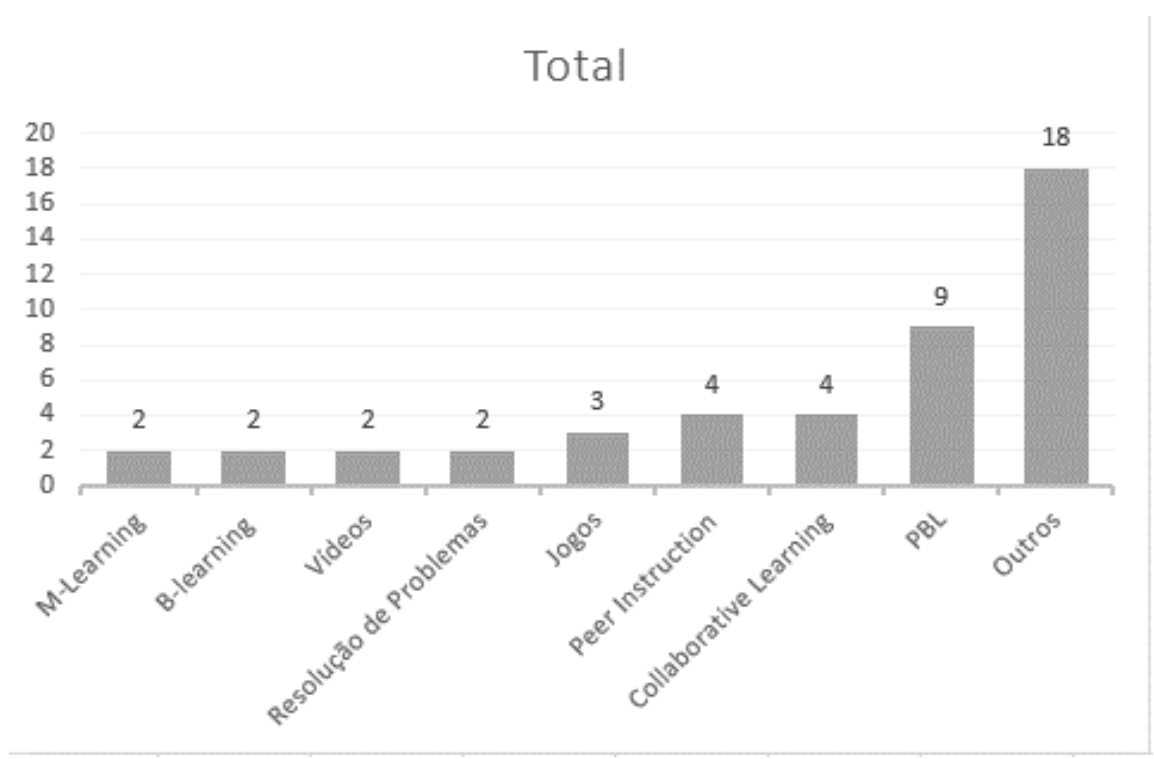

Fonte: os autores.

A PBL como metodologia ativa trabalha com a resolução de problemas, colocando o aluno como centro do processo. Angelo et al. (2014) relatam que o método PBL pode ser aplicado com sucesso ao ensino de programação de computadores. A análise revelou que os alunos demonstraram que desenvolveram a capacidade de "aprender a aprender", tanto individualmente quanto em grupo, com isso afirmando que acreditam na efetividade proposta dos autores.

Oliveira et al. (2010) trazem na conclusão três aspectos referentes ao uso da PBL que devem ser considerados: a necessidade de adaptação ao método da PBL para os tutores/ professores; comprometimento dos tutores/professores; e, por fim, a adequação da complexidade dos problemas utilizados na aplicação do método. Ainda: relatam os ganhos para os alunos, como habilidades de expressar e ouvir opiniões e tomar decisões e comunicar-se colaborativamente como pontos importantes da metodologia.

Seguindo a PBL, a Collaborative Learning e Peer Instruction foram as propostas de ensinoativas mais utilizadas. Entretanto, foi possível constatar uma variação de métodos ativos de ensino-aprendizagem, tais como: Active-Learning Exercises; Judge-based Methodology; Motivational Active Learning (MAL); Team-Based Learning; Project Based Learning; Think-PairShare e outros, conforme a Tabela 1.

Tabela 1 - Metodologias Ativas Mapeadas no Ensino de Computação

\begin{tabular}{|l|l|l|}
\hline Metodologia & Autor & Descrição \\
\hline
\end{tabular}




\begin{tabular}{|c|c|c|}
\hline $\begin{array}{l}\text { Collaborative } \\
\text { Learning }\end{array}$ & $\begin{array}{l}\text { Valdivia e Nussbaum (2007); } \\
\text { Anderson et al. (2007); } \\
\text { Hakimzadeh, Adaikkalava, } \\
\text { Batzinger (2011); } \\
\text { Lopez-guede et al. (2015) }\end{array}$ & $\begin{array}{l}\text { O aprendizado colaborativo baseia-se na visão de } \\
\text { que o conhecimento é uma construção social, em } \\
\text { que o estudante é o principal foco de instrução. } \\
\text { Utiliza-se do trabalho em grupos em abordagens } \\
\text { estruturadas para desenvolver soluções para } \\
\text { problemas do mundo real. }\end{array}$ \\
\hline $\begin{array}{l}\text { Peer-Led } \\
\text { Team } \\
\text { Learning }\end{array}$ & $\begin{array}{l}\text { Roach e Villa (2007); } \\
\text { Sperry e Tedford (2008) }\end{array}$ & $\begin{array}{l}\text { Grupos de 6-8 estudantes que trabalham juntos } \\
\text { para resolver problemas e são liderados na sua } \\
\text { resolução por um de seus pares. }\end{array}$ \\
\hline $\begin{array}{l}\text { Exercícios } \\
\text { ativos }\end{array}$ & Davis (2007) & $\begin{array}{l}\text { Criação de exercícios sobre a temática abordada } \\
\text { pelo professor e resolvida pelos alunos; forma } \\
\text { clássica de aprendizagem. }\end{array}$ \\
\hline $\begin{array}{l}\text { Problem } \\
\text { Based } \\
\text { Learning }\end{array}$ & $\begin{array}{l}\text { Chinn, Spencer e Martin } \\
\text { (2007); } \\
\text { Oliveira et al. (2010); } \\
\text { Chang e Lee (2010); } \\
\text { O'Grady (2012); } \\
\text { Arbelaitz, Martín e Muguerza } \\
\text { (2014); } \\
\text { Angelo et al. (2014); } \\
\text { Fragoso et al (2015); } \\
\text { Giannakos, Krosgstie, Aalberg } \\
\text { (2016); } \\
\text { Mourão (2017) }\end{array}$ & $\begin{array}{l}\text { Problem Based Learning trata-se de uma } \\
\text { metodologia de ensino ativa que se utiliza de } \\
\text { problemas do universo real do estudante para a } \\
\text { construção do conhecimento. São levantadas } \\
\text { hipóteses de solução, busca-se o aprendizado } \\
\text { autônomo, a resolução do problema e o } \\
\text { compartilhamento dos resultados. }\end{array}$ \\
\hline $\begin{array}{l}\text { Resolução de } \\
\text { Problemas }\end{array}$ & $\begin{array}{l}\text { Rogonis e Hazzan (2008); } \\
\text { Riek (2013) }\end{array}$ & $\begin{array}{l}\text { A metodologia de resolução de problemas consiste } \\
\text { em promover a aprendizagem ativa em que os } \\
\text { estudantes trabalham e desenvolvem o } \\
\text { conhecimento. Isso se dá com base em problemas } \\
\text { da realidade dos estudantes. }\end{array}$ \\
\hline $\begin{array}{l}\text { Scaffolding } \\
\text { Technique }\end{array}$ & $\operatorname{Jin}(2008)$ & $\begin{array}{l}\text { Método em andaime que descreve o apoio } \\
\text { adaptativo e temporário fornecido por uma pessoa } \\
\text { mais competente (por exemplo, professor ou } \\
\text { adulto) a uma pessoa menos capacitada. }\end{array}$ \\
\hline Metodologia & Autor & Descrição \\
\hline Jogos & $\begin{array}{l}\text { Anewalt (2008); } \\
\text { Papastergiou (2009); } \\
\text { Voss (2015) }\end{array}$ & $\begin{array}{l}\text { Utilização de jogos como ferramentas de ensino- } \\
\text { aprendizagem. }\end{array}$ \\
\hline $\begin{array}{l}\text { Collaborative } \\
\text { Learning } \\
\text { Communities }\end{array}$ & Anderson e Lin (2009) & $\begin{array}{l}\text { Criação de uma comunidade de aprendizagem } \\
\text { colaborativa, com a ideia de conectar estudantes } \\
\text { fora da sala de aula e, assim, melhorar a } \\
\text { aprendizagem deles por meio dessa conexão. }\end{array}$ \\
\hline
\end{tabular}




\begin{tabular}{|c|c|c|}
\hline $\begin{array}{l}\text { Judge-based } \\
\text { Methodology }\end{array}$ & $\begin{array}{l}\text { Gárcia-Mateos e Fernández- } \\
\text { Alemán (2009) }\end{array}$ & $\begin{array}{l}\text { Metodologia que se utiliza de recursos de } \\
\text { julgamento online. Os estudantes resolvem } \\
\text { problemas predefinidos e os submetem às } \\
\text { ferramentas que permitem o julgamento online. } \\
\text { Este julgamento pode ser automático, possuindo } \\
\text { entradas e saídas predefinidas, ou então um } \\
\text { sistema que permita a análise da resolução por } \\
\text { outros. }\end{array}$ \\
\hline $\begin{array}{l}\text { Active- } \\
\text { Learning } \\
\text { Exercises }\end{array}$ & Gehringer e Miller (2009) & $\begin{array}{l}\text { Os exercícios de aprendizagem ativa consistem em } \\
\text { aplicar abordagens ativas. O autor usa Think-Par- } \\
\text { Shared em atividades de sala de aula. }\end{array}$ \\
\hline $\begin{array}{l}\text { Team-Based } \\
\text { Learning }\end{array}$ & Lasserre (2009) & $\begin{array}{l}\text { Forma de aprendizagem colaborativa. Trabalha } \\
\text { com formação de equipes permanentes, aplicação } \\
\text { de atividades e avaliações em pares. }\end{array}$ \\
\hline $\begin{array}{l}\text { Sessões } \\
\text { Práticas e } \\
\text { Teóricas }\end{array}$ & $\begin{array}{l}\text { Muresano, Rexachs e Luque } \\
\text { (2010) }\end{array}$ & $\begin{array}{l}\text { A metodologia integra aulas teóricas e práticas, } \\
\text { nas quais os alunos podem aprender e praticar os } \\
\text { conceitos. }\end{array}$ \\
\hline M-learning & $\begin{array}{l}\text { Morais, Alencar e Souza } \\
(2011) \text {; } \\
\text { Lindquist et al. (2007) }\end{array}$ & $\begin{array}{l}\text { O mobile learning é uma modalidade da educação } \\
\text { a distância. Usufrui de ferramentas móveis para } \\
\text { dar suporte às atividades de ensino. }\end{array}$ \\
\hline $\begin{array}{l}\text { Inductive } \\
\text { instruction }\end{array}$ & $\begin{array}{l}\text { Moura e Van Hattum-Janssen } \\
\text { (2011) }\end{array}$ & $\begin{array}{l}\text { Metodologia que, ao invés de o professor explicar } \\
\text { um determinado conceito e seguir essa explicação } \\
\text { com exemplos, apresenta aos estudantes muitos } \\
\text { exemplos que mostram como o conceito é usado. } \\
\text { A intenção é que os estudantes "notem", por meio } \\
\text { dos exemplos, como o conceito funciona. }\end{array}$ \\
\hline $\begin{array}{l}\text { Peer } \\
\text { Instruction }\end{array}$ & $\begin{array}{l}\text { Porter, Lee e Simon (2013); } \\
\text { Grissom (2013); } \\
\text { Zingaro e Porter (2014); } \\
\text { Lima et al. (2016) }\end{array}$ & $\begin{array}{l}\text { Peer Instruction trata-se de uma metodologia que } \\
\text { tem a necessidade de que cada estudante leia, } \\
\text { pense e reflita antes da aula o assunto a ser } \\
\text { abordado. Assim, já em sala de aula, será posta a } \\
\text { problematização e discutida a temática entre os } \\
\text { pares. }\end{array}$ \\
\hline $\begin{array}{l}\text { Pedagogical } \\
\text { Code Reviews }\end{array}$ & $\begin{array}{l}\text { Hundhausen, Agrawal e } \\
\text { Agarwal (2013) }\end{array}$ & $\begin{array}{l}\text { Metodologia inspirada na inspeção do código } \\
\text { utilizado na indústria de software. Trata-se de uma } \\
\text { atividade colaborativa em que uma pequena } \\
\text { equipe de estudantes, liderada por um moderador, } \\
\text { caminha por meio de segmentos de soluções de } \\
\text { programação para cada integrante. Em que, se } \\
\text { verifica o código em relação a uma lista de } \\
\text { melhores práticas de codificação, permitindo } \\
\text { discutir e registrar os problemas que surgem no } \\
\text { desenvolvimento. }\end{array}$ \\
\hline Metodologia & Autor & Descrição \\
\hline Vídeos & $\begin{array}{l}\text { Largent (2013); } \\
\text { Silva et al. (2015) }\end{array}$ & $\begin{array}{l}\text { Método de ensino por meio de elaboração de } \\
\text { vídeos educacionais ou utilização destes como } \\
\text { instrumento de ensino (videoaula). }\end{array}$ \\
\hline
\end{tabular}




\begin{tabular}{|c|c|c|}
\hline $\begin{array}{l}\text { Tutoria/Curso } \\
\text { Introdutório }\end{array}$ & Zhang et al. (2013) & $\begin{array}{l}\text { Curso introdutório. Utiliza-se do conceito de } \\
\text { tutoria antes de abordar o assunto em sala de } \\
\text { aula. }\end{array}$ \\
\hline $\begin{array}{l}\text { Think-Pair- } \\
\text { Share }\end{array}$ & $\begin{array}{l}\text { Kothiyal et al. (2013); } \\
\text { Dol (2014) }\end{array}$ & $\begin{array}{l}\text { Think-pair-share (TPS) é uma estratégia de } \\
\text { aprendizagem colaborativa em que os estudantes } \\
\text { trabalham juntos para resolver um problema. } \\
\text { Exige que os estudantes pensem individualmente } \\
\text { sobre um tópico ou respondam a uma pergunta e } \\
\text { compartilhem ideias com os colegas de classe. } \\
\text { Dentro do processo, ao discutir com um parceiro, } \\
\text { maximizará a participação. }\end{array}$ \\
\hline $\begin{array}{l}\text { Motivational } \\
\text { Active } \\
\text { Learning } \\
\text { (MAL) }\end{array}$ & $\begin{array}{l}\text { Pirker, Riffnaller-Schiefer e } \\
\text { Gütl (2014) }\end{array}$ & $\begin{array}{l}\text { Modelo pedagógico que incorpora estratégias de } \\
\text { aprendizagem a partir de aprendizagem ativa e } \\
\text { aprendizagem colaborativa, combinada com } \\
\text { estratégias de gamificação. }\end{array}$ \\
\hline $\begin{array}{l}\text { Socially- } \\
\text { Inspired } \\
\text { Learning }\end{array}$ & Burh et al. (2015) & $\begin{array}{l}\text { Proposta de aprendizagem dentro de uma } \\
\text { comunidade e para uma comunidade. }\end{array}$ \\
\hline $\begin{array}{l}\text { Test Driven } \\
\text { Development } \\
\text { (TDD) }\end{array}$ & $\begin{array}{l}\text { Souza-Concilio, Corrêa e } \\
\text { Pacheco (2015) }\end{array}$ & $\begin{array}{l}\text { Metodologia de Desenvolvimento Orientado a } \\
\text { Testes, do inglês Test Driven Development (TDD). } \\
\text { A metodologia TDD consiste em um conjunto de } \\
\text { técnicas que encoraja projetos simples e conjuntos } \\
\text { de testes com o intuito de agregar confiança, de } \\
\text { acordo com Souza-Concilio, Corrêa e Pacheco } \\
\text { (2015). Há duas regras básicas que precisam ser } \\
\text { seguidas: primeiro, deve-se escrever um teste } \\
\text { automatizado que falhe antes de escrever algum } \\
\text { código; a outra regra diz que se deve remover } \\
\text { código duplicado. }\end{array}$ \\
\hline B-learning & $\begin{array}{l}\text { Gonzáles (2013); } \\
\text { Olelewe e Agomuo (2016) }\end{array}$ & $\begin{array}{l}\text { O blended learning consiste na junção dos } \\
\text { conceitos de aula e-learning, ensino online a } \\
\text { distância, com o modelo presencial. Assim, trata- } \\
\text { se de uma proposta mista de ensino. }\end{array}$ \\
\hline
\end{tabular}

Fonte: os autores.

Entre os resultados supracitados, a aprendizagem colaborativa, na qual o conhecimento surge devido ao envolvimento e à contribuição ativa dos estudantes, dialoga com as metodologias ativas. A metodologia Peer Instruction é um exemplo. Esta possui dois objetivos básicos, conforme apontam Araujo et al. (2016). Quais sejam: explorar a interação entre os estudantes e trabalhar com problemas propostos em sala, promovendo uma aprendizagem colaborativa.

Dentro das metodologias colaborativas e ativas, observa-se também a Peer-Led Team Learning (PLTL), definida por Roach e Villa (2007) como uma proposta que promove a interdependência positiva, individual e responsabilização em grupo; além disso, aprimora as habilidades sociais. Snyder et al. (2016) a definem como um modelo de aprendizagem ativo 
bem definido, envolvendo pequenas interações entre os estudantes, podendo ser usada em conjunto com o formato tradicional de palestras.

As propostas que empregaram vídeos e jogos foram classificadas como ativas devido à forma de aplicação. Largent (2013) propõe que os estudantes construam videoaulas como tática de ensino. Anewalt (2008), Papastergiou (2009) e Voss (2015) usam a gamificação como uma abordagem ativa.

Já os exercícios de aprendizagem ativa (Active-Learning Exercises), listados como metodologia ativa, consistem em abordagens ativas em sala de aula, a partir das quais, de acordo com Gehringer e Miller (2009), os estudantes realizam atividades ativas com base em problemas dados pelo professor, de modo que a resolução lhes permita descobrir novos conhecimentos ou ampliar os conhecimentos existentes, utilizando recursos escolhidos pelos próprios aprendizes.

A metodologia Think-Pair-Share (TPS) pode ser empregada como uma metodologia ativa ou ainda como um método dentro dos Active-Learning Exercises. Conforme explica Dol (2014), o próprio acrônimo do nome é dividido em três etapas: think, cuja finalidade é apresentar a problemática do tema; pair, na qual os envolvidos se dividem em pares e discutem as soluções para a problemática; share, na qual as soluções são trazidas ao grande grupo, sendo compartilhadas com a turma.

Outro exemplo de metodologia ativa aplicada à computação é descrito por Gárcia-Mateos e Fernández-Alemán (2009), denominado Judge-based Methodology. De acordo com os estes autores, consiste em utilizar o sistema de julgamento online para avaliar a solução de problemas desenvolvidos pelos estudantes.

Hundhausen, Agrawal e Agarwal (2013) apresentam outra metodologia ativa para o ensino de computação: Pedagogical Code Reviews. Essa metodologia, de acordo com estes autores, surgiu inspirada no método de inspeção de código de área de engenharia de software. A proposta consiste em trabalhar com uma pequena equipe de estudantes, liderada por um moderador treinado que deve percorrer segmentos das soluções de programação de outro aluno e verificar o código, para isso seguindo as melhores práticas de codificação.

As obras apontam várias características referentes às metodologias ativas. Um grande foco está no protagonismo do aprendiz em sala de aula, na sua independência e na autonomia dentro do processo, mas também na colaboração e no compartilhamento de conhecimento com os demais alunos.

Com base nesses 46 artigos selecionados, foi possível identificar as áreas de ensino de computação nas quais foram empregadas as metodologias ativas (MA). Esse resultado comprovou um grande foco voltado para a área de programação, como pode ser observado na Figura 4. 


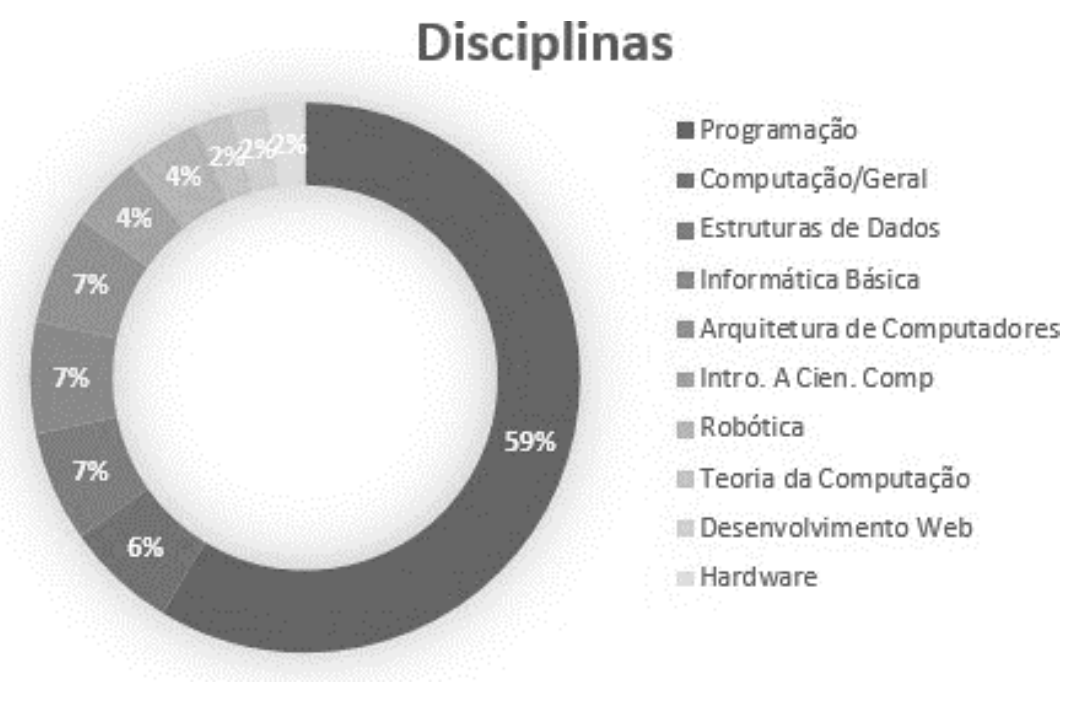

Fonte: os autores.

O ensino de programação representa o maior enfoque na utilização de metodologias ativas. De acordo com Piekarski et al. (2015), o conteúdo de programação é considerado um dos mais complexos na área de computação, sendo uma das bases mais importantes para a futura atuação profissional dos estudantes. Para Mourão (2017), o grau de dificuldade dessa área se reflete nos índices de desistências e no alto índice de reprovação.

Com esse pensamento, Souza et al. (2016) elaboraram uma revisão sistemática, identificando dentro das literaturas os problemas com relação às dificuldades do ensino de programação. Nos resultados obtidos, observou-se que os problemas relacionados à aplicação desses conceitos de programação estão na construção de programas e na motivação. Dessa forma, a aprendizagem ativa, na sua essência, se encaixa como alternativa solucionadora para o aprendizado.

Isso pode ser observado na proposta de Gehringer e Miller (2010), que, para o ensino de programação, utiliza o método de Active-Learning Exercises, apresentando uma adaptação da metodologia e colocando os alunos na elaboração dos exercícios ativos a serem trabalhados, tanto na sua correção quanto na organização. Com isso, adapta-se o papel de professor, passando o aluno a ter um papel central. Ao final, os autores relatam a experiência de que, com a utilização da aprendizagem ativa, os alunos estavam mais atentos às aulas, sendo um dos pontos-chave da sua aplicação, além do sucesso de que os mesmos trabalharam para suprimir suas deficiências colaborativamente. Entre os processos de avaliação discente que estavam sendo utilizados, constatou-se que a forma de avaliação não teve uma variação das metodologias tradicionais.

Na Figura 5, os resultados das produções apontam que, em sua maioria, na forma de avaliação discente, os autores utilizavam avaliações no formato tradicional, mesmo que no processo de ensino-aprendizagem tenha sido utilizada uma metodologia ativa. 


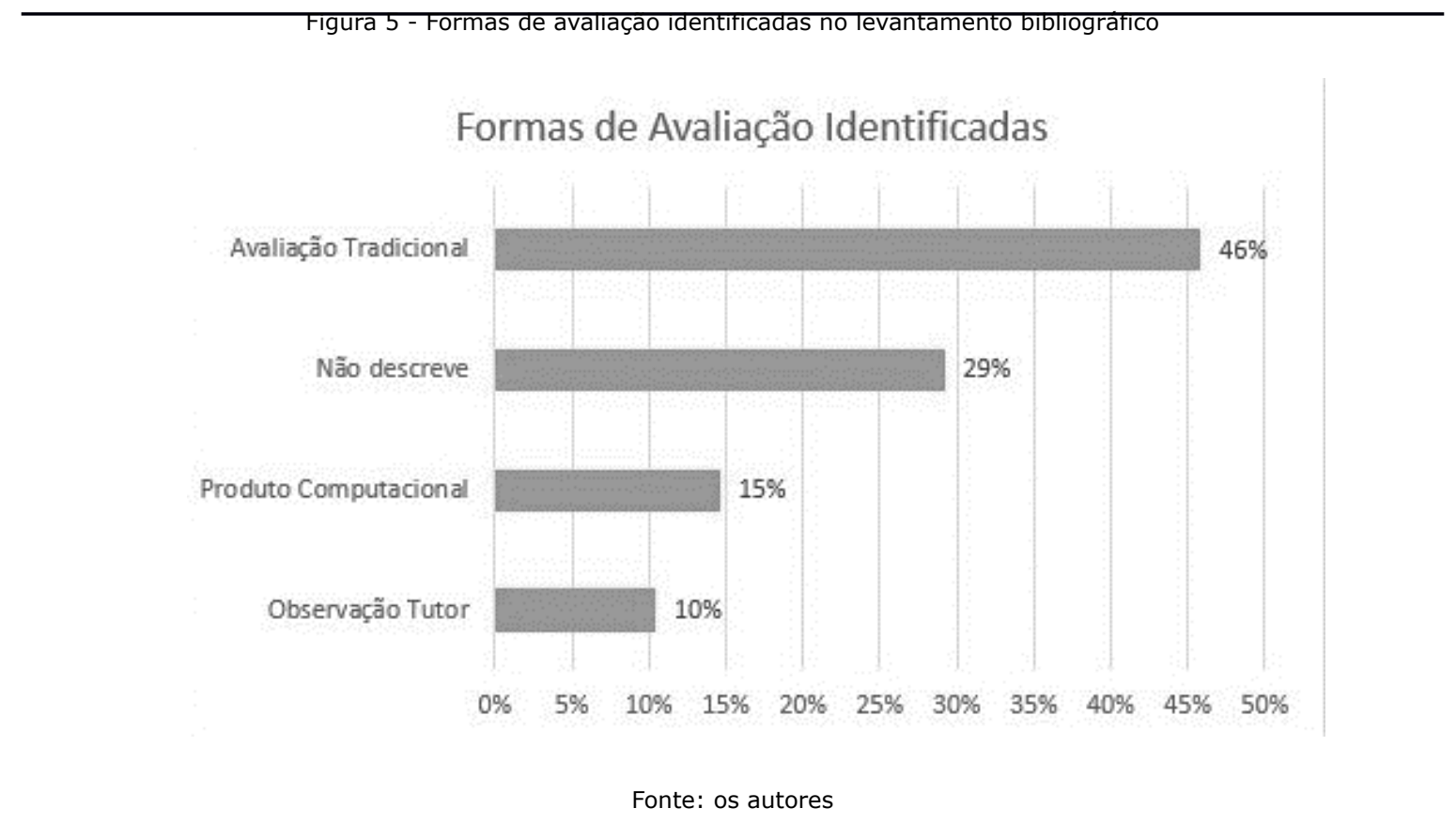

Em nossa análise, consideramos tradicional a avaliação fazendo uso de provas teóricas de conhecimento e destacando a utilização de testes de conhecimento, que podem ser vistos em Angelo et al. (2014); Isomottonen e Tirronen (2013); Oliveira et al. (2010); Valdivia e Nussbaum (2017); Chang e Lee (2010); Anderson e Lin (2009); Hundhausen et al. (2013); Zhang et al. (2013); Olelewe e Agomuo (2016); Mourão (2017); Gonzáles et al. (2013); Jin (2008); Lassere (2009); Porter e Lee (2013); Dol (2014); Papastergiou (2009).

Conforme a Figura 6, percebe-se a utilização de formação de grupos como uma estratégia didático-pedagógica dentro das metodologias ativas de aprendizagem. Isso se dá devido ao processo de ensino-aprendizagem colaborativo, que é reforçado e mais utilizado dentro das propostas apresentadas. O segundo instrumento que apareceu em maior número foi a realização de exercícios, sendo esta uma abordagem tradicional de ensino, principalmente como forma de avaliação. 
FIgura 6 - Instrumentos de avaliaçao utilizados

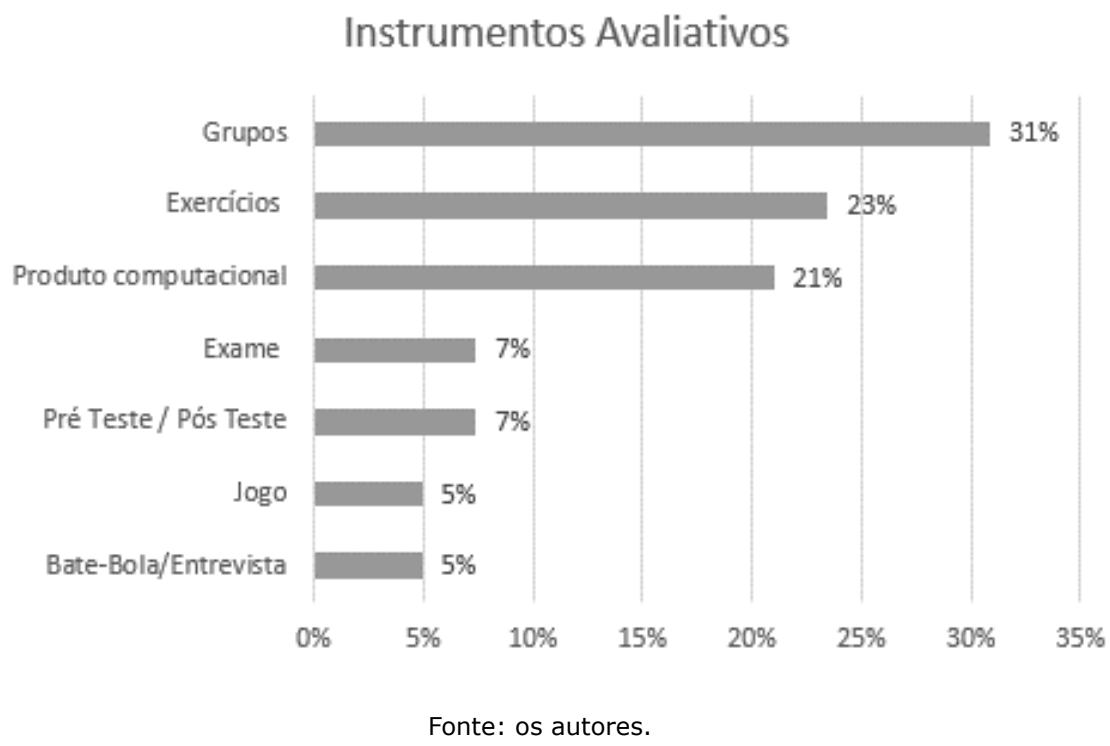

Entre os instrumentos de avaliação empregados, foi possível observar o desenvolvimento de um produto computacional, visto como uma forma de avaliação não tradicional adotada pelo pesquisador (avaliação escrita), mas utilizado como um instrumento de obtenção de nota. Trata-se de algo que corresponde à atividade de criação de um software, uma rotina, uma codificação, a fim de resolver um problema determinado ou um relatório de arquitetura de um sistema. A técnica de construção de um produto computacional é uma abordagem comum dentro do ensino de computação, sendo um resultado esperado como processo de avaliação do aluno.

Dentro do contexto de avaliação, uma das técnicas que se destaca é a aplicação de prétestes e pós-testes para a quantificação do aprendizado na utilização da metodologia, conforme observadas em Papastergiou (2009); Zhang et al. (2013); Dol (2014); Hundhausen et al. (2013); Gonzáles et al. (2013); Olelewe e Agomuo (2016). O emprego desses pré-testes e pós-testes é uma técnica na qual se aplica um teste do que se deseja medir, antes e depois da aplicação. Por exemplo, um teste de conhecimento prévio, no qual, após a execução do processo, é feito um novo teste com o intuito de compara-lo ao teste inicial, permitindo a observação de dados comparativos entre as situações inicial e final da pesquisa.

Nos resultados obtidos, é possível destacar uma das técnicas de avaliação, cuja proposta consiste na ideia de uma entrevista a partir da qual a medição do conhecimento se dá pela verificação do diálogo com o professor. A proposta nesse aspecto que chamou atenção foi a técnica apresentada por Oliveira et al. (2010), denominada "bate bola", na qual, além de uma avaliação tradicional, também se aplica uma avaliação sobre o produto computacional e sobre os conhecimentos mensurados segundo a percepção do professor. Isso se dá por meio de uma conversa com o aluno.

Dentro da questão avaliativa, no processo de avaliação da metodologia, constatou-se que o instrumento mais utilizado foi a realização de questionários, conforme pode ser observado na Figura 7. 


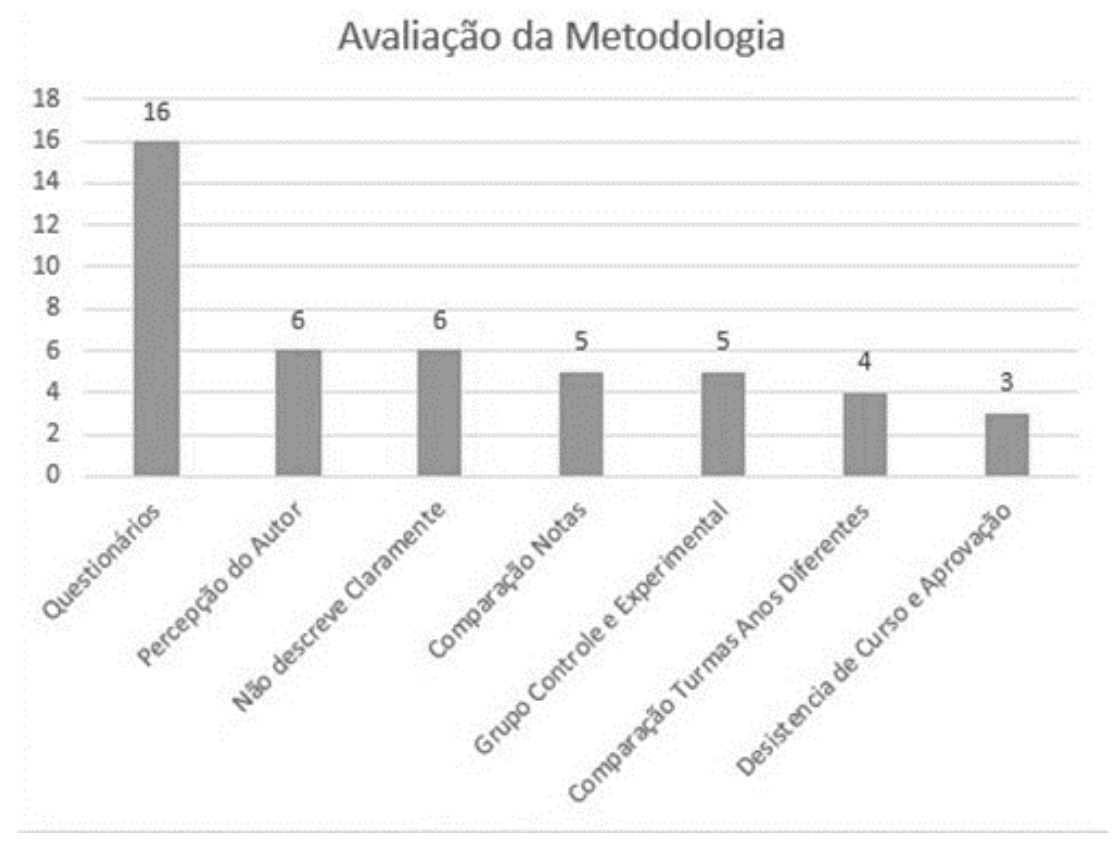

Fonte: os autores.

O questionário aparece como um instrumento que permite uma forma mais acessível de coleta de dados, sendo o mais empregado na maioria dos casos. O intuito de sua aplicação nesse cenário foi de validação da metodologia como uma proposta para o ensino de computação. Esses questionários, em sua maioria, foram aplicados aos alunos, dando-lhes voz e identificando a percepção do estudante quanto à utilização da metodologia.

Angelo et al. (2014), quanto à proposta de questionário, apresentam questões que deixam claro a abordagem da validação da metodologia, como na questão "Como você avalia seu aprendizado durante as sessões de tutoriais e os estudos com os colegas?". Esse aspecto é medido por meio de uma questão fechada que apresenta as alternativas: péssimo, ruim, bom, muito bom e ótimo. Para esse caso, o resultado da classificação pelos alunos foi "bom".

O resultado indicou que os alunos perceberam o aprendizado deles em uma proposta colaborativa. Porém, no que se refere ao processo de aprendizagem extraclasse e às tutorias, Angelo et al. (2014, p. 36) explicam que o resultado "parece indicar que a efetividade do método, para os alunos, está mais associada às sessões tutoriais do que ao aprendizado extraclasse, embora este seja um dos momentos principais do método".

Ainda buscando identificar a percepção dos alunos quanto à metodologia empregada, Angelo et al. (2014) fazem a seguinte pergunta: "Você acredita que a aplicação do método PBL no Estudo Integrado de Programação foi efetiva para aprender o que você aprendeu?". Como possibilidade de resposta, apresentou-se as seguintes opções: não acredito, acredito parcialmente ou acredito totalmente. Nessa questão, a maioria dos estudantes informou que acredita parcialmente. Assim, de acordo com o Angelo et al. (2014), o resultado mostra a insegurança dos alunos quanto a percepção do próprio aprendizado, correspondendo a um ponto a ser melhorado no futuro. 
Com base nos resultados encontrados na pesquisa, é possível compreender a abordagem das metodologias ativas e seus impactos no ensino de computação. É igualmente possível identificar a diversidade de metodologias que apoiam de forma ativa o processo ensinoaprendizagem de alunos e professores, e que estão sendo aplicadas e avaliadas como propostas aos desafios encontrados por professores e pesquisadores da área de computação.

\section{Considerações finais}

O ensino de computação está em busca de novas metodologias que permitam o desenvolvimento do processo de aprendizagem. A metodologia ativa, nesse aspecto, vem se tornando cada vez mais uma alternativa viável para a área em relação à metodologia tradicional que ainda vem sendo empregada.

A pesquisa mostrou como resultado um interesse claro em explorar os métodos ativos de aprendizagem, em especial focado no ensino de programação, que hoje é uma das áreas com maior histórico de reprovação.

Como resultado, o artigo revelou que, entre as abordagens ativas identificadas, constatouse o interesse no uso da PBL (Problem Based Learning). A PBL é apontada como uma das metodologias mais utilizadas entre as abordagens ativas empregadas no ensino de computação.

Observou-se uma variedade de metodologias ativas, tais como: Collaborative Learning Communities; Judge-based Methodology; Pedagogical Code Reviews; Test Driven Development; Think-Pair-Share; Active Learning Exercises entre outras. Essa variedade mostra o interesse dos professores e pesquisadores pelo estudo e busca de uma metodologia eficaz para os problemas no ensino de computação, a exemplo das dificuldades de aprendizagem devido à complexidade do assunto, abstração de problemas, alto índice de reprovação nos cursos, entre outros.

Mesmo com o interesse em abordar o desenvolvimento de uma proposta centrada no aluno, a pesquisa mostrou que, na avaliação dos estudantes, os autores adotaram uma abordagem que vai ao encontro a proposta tradicional, com a aplicação de testes, provas de conhecimento ou exames, como pôde ser observado em Angelo et al. (2014); Isomottonen e Tirronen (2013); Oliveira et al. (2010); Valdivia e Nussbaum (2017); Chang e Lee (2010); Anderson e Lin (2009); Zhang et al. (2013); Olelewe e Agomuo (2016); Mourão (2017); Gonzáles et al. (2013); Jin (2008); Lassere (2009); Porter e Lee (2013); Dol (2014); Papastergiou (2009).

Percebe-se que o processo de avaliação com base em testes não foi descartado pelos profissionais da área, mas, sim, aliado à outras abordagens, a exemplo da avaliação "bate bola", produção de vídeos, desenvolvimento computacional, entre outras. Assim, como resultado, identificou-se um campo a ser discutido e estudado a fim de gerar propostas alternativas quanto à aplicação de uma avaliação formal, no sentido de que, ao utilizar uma metodologia que foge dos padrões tradicionais, o processo de avaliação deveria seguir uma proposta que se ajuste à essa ideologia.

Com relação às limitações e às ameaças a esta pesquisa, é cabível destacar neste trabalho o intervalo de anos de pesquisa - de 2007 a 2018 - e a quantidade de MBAs utilizados. Tais 
limitações, mesmo que necessárias para a condução de qualquer pesquisa, podem deixar de retratar a realidade mundial do tema pesquisado. Dessa maneira, de acordo com as restrições à esta investigação e como sugestão para estudos futuros, recomenda-se que as próximas pesquisas nessa área devem considerar um número maior de MBAs e utilizar processos formais e validados de mapeamentos sistemáticos, expandindo, assim, o número de possíveis trabalhos a serem analisados, bem como atualizar o período de busca, incrementando estudos mais recentes.

Ainda em termos de futuros trabalhos, os pesquisadores veem como uma área de interesse a aplicação híbrida entre metodologias ativas, reforçando a aprendizagem ativa pelo estudante. Igualmente veem a criação ou instrumentalização dessas propostas, auxiliando o professor no emprego dessas metodologias em sala de aula.

\section{Referências}

ARAUJO, A. V. R. de et al. Uma associação do método Peer Instruction com circuitos elétricos em contextos de aprendizagem ativa. Revista Brasileira de Ensino de Física, [s.I.], v. 39, n. 2, 7 nov. 2016. FapUNIFESP (SciELO). Disponível em: <http://dx.doi.org/ 10.1590/1806-9126-rbef-2016-0184>. Acesso em: 10 abr. 2018.

ARBELAITZ, O.; MARTÍN, J. I.; MUGUERZA, J.Analysis of Introducing Active Learning Methodologies in a Basic Computer Architecture Course. IEEE Transactions on Education. 2014. Disponível em:<http:// ieeexplore.ieee.org /document/ 6851215/>

ANDERSON, R. et al. Supporting active learning and example based instruction with classroom technology. SIGCSE, 2007. Disponível em: https://dl.acm. org/citation.cfm?id=1227338

ANDERSON, N.; LIN, C.-C. Exploring technologies for building collaborative learning communities among diverse student populations. ITiCSE '09 Proceedings of the 14th annual ACM SIGCSE conference on Innovation and technology in computer science education, páginas 243-247. 2009.

ANGELO, M. F. et al. Aplicação e avaliação do método pbl em um componente curricular integrado de programação de computadores. Revista de Ensino de Engenharia (2), páginas 31-43. ISSN 2236-0158. 2014.

ANEWALT, K. Making CSO fun: an active learning approach using toys, games and Alice. Journal of Computing Sciences in Colleges. 2008. Disponível em: <https://dl.acm.org/ citation.cfm?id=1295133>

BARBOSA, E. F.; MOURA, D. G. Metodologias ativas de aprendizagem no ensino de engenharia. XIII International Conference on Engineering and Technology Education(1), páginas 16-19. ISSN 2317-4382. 2014.

BELLAN, Z. S. Andragogia em ação: Como ensinar adultos sem se tornar maçante. SOCEP Editora, no 3. Santa Bárbara d'Oeste, SP. pags. 156. ISBN 8598486167. 2008.

BERBEL, N. A. N. As metodologias ativas e a promoção da autonomia de estudantes. Semina: Ciências Sociais e Humanas, 32(1), página 25-40. 2011.

BERNHEIM, C. T; CHAUI, M. S. Desafios da Universidade na Sociedade do Conhecimento: cinco anos depois da Conferencia sobre ensino superior. Brasília: UNESCO, 2008. Disponível em: https:// unesdoc.unesco.org /ark:/48223/pf0000134422_por. Acesso em: 12 fev. 2018.

BISHOP, J.; VERLEGER, M. The flipped classroom: A survey of the research. In ASEE National Conference Proceedings. 2013. 
BUCHINGER, D; CAVALCANTI, G. A. de S.; HOUNSELL, M da S. 2014. Mecanismos de busca acadêmica: uma análise quantitativa. Revista Brasileira de Computação Aplicada, [s.I.], v. 6, n. 1, p.108-120, 28 abr. UPF Editora. 2014.

$B U R H$, J. et al. Engaging non-traditional students in computer science through socially-inspired learning and sustained mentoring. 46th ACM Technical Symposium on Computer Science Education. 2015. Disponível em: <https://dl.acm.org/citation.cfm? id=2677266>

CHANG, L.-C.; LEE, G. A team-teaching model for practicing project-based learning in high school: Collaboration between computer and subject teachers. Computers And Education, 55(3), páginas 961-969. ISSN 0360-1315. 2010.

CHINN, D.; SPENCER, C.; MARTIN, K. 2007. Problem solving and student performance in data structures and algorithms. 12th Annual SIGCSE Conference on Innovation and Technology in Computer Science Education. 2007. Disponível em: <https://dl.acm.org/citation.cfm? id=1268854>

DAVIS, J.; rebelsky, S. A. Food-First Computer Science: Starting the First Course Right with PB\&J. 38th SIGCSE Technical Symposium on Computer Science Education. Kentucky, USA. 2007. Disponível em: <https://dl.acm.org/ citation.cfm?i d=1227440>

DOL, S. M. TPS (think-pair-share): an active learning strategy to teach theory of computation course. International Journal of Educational Research and Technology, 5(4), páginas 62-67. ISSN 0360-1315. doi: 10.15515. 2014

FONSECA, S. M.; MATTAR NETO, J. A.. Metodologias ativas aplicadas à educação a distância: revisão de literatura. Revista Edapeci, São Cristóvão (se), v. 17, n. 2, p.185-197, ago. 2017.

FRAGoSo, M. L. P. G. et al. Viabilizando uma Produção de Obra de Arte Robótica por Meio de Metodologia de Aprendizagem Baseada em Projetos. ART Encontro Internacional de Arte e Tecnologia - Brasília DF. 2012. Disponível em: <https://art.medialab.ufg. br/up/779/o/JuliaZ-all.pdf>

GÁRCIA-MATEOS, G., FERNÁNDEZ-ALEMÁN, J. L. A course on algorithms and data structures using on-line judging. 14th annual ACM SIGCSE conference on Innovation and technology in computer science education. 2009. Disponível em:<https://dl.acm.org/citation.cfm?id=1562897>

GARAY, G. R. et al. Visualization of VHDL-based simulations as a pedagogical tool for supporting computer science education. Journal of Computational Science. 2017. Disponível em: <http://www.sciencedirect.com /science/article/pii/S187775031730385X>

GEHRINGER, E. F.; MILLER, C. S. Student-generated active-learning exercises. SIGCSE '09: Proceedings of the 40th ACM technical symposium on Computer science education, 41(1), páginas 81-85. ISBN 978-1-60558-183-5. 2009.

GIL, A. C. Metodologia do Ensino Superior. 3. ed. - São Paulo: Atlas, 1997.

GIL, A. C. Métodos e técnicas de pesquisa. 6 ed. Editora Atlas. São Paulo - SP. ISBN 8522451427. 2008.

GONZÁLES, A.-B. et al. Experimental evaluation of the impact of b-learning methodologies on engineering students in Spain. Computers in Human Behavior, 29(2), páginas 370-377. ISSN 0747-5632. 2013.

GRABINGER, R.; DUNLAP, J. Rich environments for active learning: A definition. Association for Learning Technology Journal, 3(2), páginas 5-35. 1995.

GRISSOM, S. Introduction to special issue on alternatives to lecture in the computer science classroom. ACM Transactions on Computing Education (TOCE). 2013. Disponível em: <https://dl.acm.org/ citation.cfm ?id=2499948>

HAKIMZADEH, H.; ADAIKKALAVAN, R.; BATZINGER, R. Successful implementation of an active learning laboratory in computer science. 39th annual ACM SIGUCCS conference on User services (SIGUCCS '11). 2011. Disponível em:<https:// dl.acm.org/citation.cfm?id =2070386> 
HUNDHAUSEN, C. D., AGRAWAL, A.; AGARWAL, P. Talking about code: Integrating pedagogical code reviews into early computing courses. ACM Transactions on Computing Education. 2013.

ISOMOTTONEN, V.; TIRRONEN, V. Teaching programming by emphasizing self-direction: How did students react to active role required of them? ACM Transactions on Computing Education (TOCE), 13(2). 2013.

JIN, W. Pre-programming analysis tutors help students learn basic programming concepts. SIGCSE '08 Proceedings of the 39th SIGCSE technical symposium on Computer science education, 40(1), páginas 276-280. 2008.

KANE, L. Educators, learners and active learning methodologies. International Journal of Lifelong Education, 23(21), páginas 275-288. 2004.

LARGENT, D. L. Flipping a large CSO course: an experience report about exploring the use of video, clickers and active learning. Journal of Computing Sciences in Colleges.2013. Disponível em:<https://dl.acm.org/ citation.cfm?id $=2527164>$

LASSERRE, P. Adaptation of team-based learning on a first term programming class. ITiCSE '09 Proceedings of the 14th annual ACM SIGCSE conference on Innovation and technology in computer science education, 41(3), páginas 186-190. 2009.

LINDQUIST, D. et al. Tabela1 Exploring the potential of mobile phones for active learning in the classroom. 38th IGCSE technical symposium on Computer science education, pag. 384-388. 2007. Disponível em:<https:// dl.acm.org/ citation.cfm?id=1227445>

LIMA, S. J. S. De, et al. "Aplicação de uma Metodologia Ativa para o Ensino de Lógica de Programação". Anais do EATI - Encontro Anual de Tecnologia da Informação e STIN - Simpósio de tecnologia da Informação da Região Noroeste do RS, Frederico Westphalen - RS, Ano 6 n. 1 p. 209-212. 2016. Disponível em:<http://eati. info/eati/2016/assets/anais/Curtos/209.pdf>

LOPEZ-GUEDE, J. M. et al. Educational Innovation in the Computer Architecture Area.Procedia - Social and Behavioral Sciences.2015.Disponível em:<http://www.sciencedirect.com/science/article/pii/S1877042 $81502354 X>$

MARIN, M.J et. al. Aspectos das fortalezas e fragilidades no uso das Metodologias Ativas de Aprendizagem. Revista brasileira de educação médica. p. 13 - 20. 2010.

MITRE, S. M. E. A. Metodologias ativas de ensino aprendizagem na formação profissional em saúde: Debates atuais. Ciência Saúde Coletiva, 13(2).2008.

MIZUKAMI, M. d. G. N. Ensino: as abordagens do processo. São Paulo: EPU, 1986. 119 p. (Temas básicos de educação e ensino).1986.

MORAIS, D. C. S. de; ALENCAR, A. D. P. C.; SOUZA, R. de. Jogo baseado em m-learning e aprendizado tangencial para auxílio ao ensino de Teoria da Computação. XXII Simpósio Brasileiro de Informática na Educação. 2011. Disponível em:<http://www.br-ie.org/pub/index.php/sbie /article/view/1838/1600>

MORAN, J. Mudando a educação com metodologias ativas. Coleção Mídias Contemporâneas. Convergências Midiáticas, Educação e Cidadania: aproximações jovens. UEPG - Universidade Estadual de Ponta Grossa, 2, páginas 15-33. 2015.

MOURA, I.C.; HATTUM-JANSSEN, N. van. Teaching a CS introductory course: An active approach. Computers \& Education. 2011.Disponível em: <http://www.sciencedirect .com/science/article /pii/ S0360131510002617>

MOURÃO, A. B. Uma proposta da eficiência do uso da metodologia ativa baseada em problemas, utilizando dojo de programação. Anais do VI Congresso Brasileiro de Informática na Educação (CBIE). Anais do XXIII Workshop de Informática na Escola (WIE), páginas 667-676. 2017. 
MURESANO, R.; REXACHS, D.; LUQUE, E. Learning parallel programming: a challenge for university students. Procedia Computer Science. 2010. Disponível em:<http://www.sciencedirect.com/ science/ article/pii/S1877050910000979>

O'GRADY, M. J. Practical problem-based learning in computing education. ACM Transactions on Computing Education, vol. 12, no. 3. 2012.Disponível em:<https://dl.acm. org/citation.cfm?id=2275599>

OLELEWE, C. J.; AGOMUO, E. E. Effects of B-learning and F2F learning environments on students' achievement in QBASIC programming. Computers \& Education.2016. Disponível em: <http://www sciencedirect .com/science/article/pii/S0360131516301750>

OLIVEIRA, W. L. A. D. et al. Aplicando pbl no ensino de arquitetura de computadores. Congresso Internacional PBL. São Paulo, Brasil. 2010.

PAIVA, M. R. F. et al. Metodologias Ativas De Ensinoaprendizagem: Revisão Integrativa. Sanare, Sobral, v. 15, n. 2, p.145-153. 2016.

PAPASTERGIOU, M. Digital game-based learning in high school computer science education: Impact on educational effectiveness and student motivation. Computers and Education, 52(1), páginas 1-12. ISSN 0360-1315. 2009.

PIAGET, J. Para onde vai a educação? 20. ed. Rio de Janeiro: J. Olympio, 127 páginas. 2011.

PIEKARSKI, A. E. T.; MIAZAKI, M. e. a. A metodologia das maratonas de programação em um projeto de extensão: Um relato de experiência. Anais dos Workshops do IV Congresso Brasileiro de Informática na Educação 2015, páginas 1246-10254. 2015.

PINTO, A. S. S., BUENO, M. R. P., SILVA, M. A. F. A., SELLMAN, M. Z.; KOEHLER, S. M. F. Inovação didática - projeto de reflexão e aplicação de metodologias ativas de aprendizagem no ensino superior: uma experiência com "peer instruction". JANUS(15), páginas 75-87. 2012.

PIRKER, J.; RIFFNALLER-SCHIEFER, M.; GUETL, C. Motivational Active Learning: Engaging University Students in Computer Science Education. Proceedings of the 2014 conference on Innovation \& technology in computer science education (ITICSE'14). pp. 297-302. 2014. Disponível em: <https://dl.acm.org/ citation. $\mathrm{cfm}$ ? id=2591750>

PORTER, L.; LEE, B., C.; SIMON. Halving fail rates using peer instruction: A study of four computer science classes. SIGCSE '13 Proceeding of the 44th ACM technical symposium on Computer science education, páginas 187-182. 2013.

RAMOS, V. et. al. A comparação da realidade mundial do ensino de programação para iniciantes com a realidade nacional: Revisão sistemática da literatura em eventos brasileiros. Anais do XXVI Simpósio Brasileiro de Informática na Educação (SBIE), Maceió, AL, Brasil, páginas 318-327. 2015.

RIEK, L. D. Embodied Computation: An Active-Learning Approach to Mobile Robotics Education, IEEE Trans. Educ. 2013. Dispoível em:<http:// ieeexplore.ieee.org/abstract/document/6341863/>

ROCHA, H. M.; LEMOS, W. M. Metodologias ativas: do que estamos falando? Base conceitual e relato de pesquisa em andamento. In.: Simpósio Pedagógico E Pesquisa Em Comunicação, 9, 2014. Rio de Janeiro: SIMPED, 2014.

ROGONIS, N.; HAZZAN, O. Tutoring Model for Promoting Teaching Skills of Computer Science Prospective Teachers. 13th Annual Conference on Innovation and Technology in Computer Science Education. 2008. Disponível em:<https://dl.acm.org/citation.cfm?id=1384345>

SILVA, T. R. et al. Investigando dois formatos de videoaulas de programação de jogos digitais para alunos do ensino médio. XXI Workshop de Informática na Escola (WIE), p.187- 196. 2015. Disponível em: <http://www.br-ie.org/pub/index.php/wie/article/view/5019/3429> 
SILVA, K. dos S.; PEREIRA, N. P.; ODAKURA, V. Mapeamento Sistemático: estratégias para o ensinoaprendizagem do Pensamento Computacional no Brasil. Nuevas Ideas En Informática Educativa, Santiago de Chile, v. 14, p.319-329, dez. 2008.

SNYDER, J. J. et al. Peer-Led Team Learning Helps Minority Students Succeed. Plos Biology, [s.I.], v. 14, n. 3, 9 mar. 2016. Public Library of Science (PLoS). Disponível em: <http://dx.doi.org/10.1371/ journal.pbio.1002398>. Acesso em: 12 out. 2017.

SOBRAL, F. R.; CAMPOS, C. J. G. Utilização de metodologia ativa no ensino e assistência de enfermagem na produção nacional: revisão integrativa. Rev. esc. enferm. USP, São Paulo, 46(1), páginas 208-218. 2012.

SOUZA-CONCILIO, I. de A.; CORRÊA, A. G. D.; PACHECO, B. de A. Coding Dojo como Metodologia de Ensino: A Visão Dos Educadores. Educação \& Tecnologia. UTFPR - Curitiba. ISSN Eletrônico 2179-6122. Ed. no 13. 2015. Disponível em: <http://revistas.utfpr. edu.br/pb/index.php/revedutec-ct/article/ view/1797>

SOUZA, D. M. D. et al. Problemas e dificuldades no ensino e na aprendizagem de programação: um mapeamento sistemático. Revista Brasileira de Informática na Educação, 24(1), páginas 39-52. 2016.

SPERRY, R. A.; TEDFORD, P. Implementing peer-led team learning in introductory computer science courses. Journal of Computing Sciences in Colleges. 2008. Disponível em: <https://dl.acm.org / citation.cfm?id=1352390>

VALDIVIA, R.; NUSSBAUM, M. Face-to-face collaborative learning in computer science classes. International Journal of Engineering Education, pag. 434-442. 2017. Disponível em: <https://www. researchgate.net / profile /Ricardo_Valdivia3/publication/228957058_Face-to-face_collaborative_learning_in _computer _science_classes/links/0f317531749fd8c5c8000000.pdf $>$.

Voss, G.; FERREIRA, C.; DENARDI, R.; POZZEBON, R. Proposta de um Jogo Educacional para o Ensino de Estrutura de Dados. Anais dos Workshops do Congresso Brasileiro de Informática na Educação (CBIE). 2015. Disponível em: <http://www.br-ie.org/pub/index.php/wcbie/article/view/6271>

WITT, D. T.; KEMCZINSKI, A.; SANTOS, L. M. D. Resolução de problemas: Abordagens aplicadas no ensino de computação. In: Computer on the beach, 2018, Florianópolis. Anais do Computer on the Beach 2018. Florianópolis: Universidade do Vale do Itajaí, 2018.

ZHANG, Z. et al. Teaching Introductory Programming to IS Students: The Impact of Teaching Approaches on Learning Performance. Journal of Information Systems Education. 2013. Disponível em: <https://www. researchgate.net/publication/261213953_Teaching_Introductory_Programming_to_IS_Students_ The_Impact_of_Teaching_Approaches_on_Learning_Performance>

ZINGARO, D.; PORTER, L. Peer Instruction in computing: The value of instructor intervention. Computers \& Education. pag 87-96. vol 71. 2014. Disponível em:<https://www. sciencedirect .com/science/article/pii/ S0360131513002777?via\%3Dihub>

Recebido em fevereiro de 2019.

Aprovado para publicação em fevereiro de 2020.

\section{Diego Teixeira Witt}

Programa de Pós-Graduação em Ensino de Ciências, Matemática e Tecnologias - Universidade do Estado de Santa Catarina - UDESC, Brasil. Professor de Informática do Instituto Federal Catarinense - IFC, Brasil. diego.witt@ifc.edu.br

\section{Avanilde Kemczinski}

Professora Associada da Universidade do Estado de Santa Catarina - UDESC, Brasil. Atua nos Programa de Pós-Graduação em Computação Aplicada - PPGCA e no Programa de Pós-Graduação em Ensino de Ciências, Matemática e Tecnologias - PPGECMT.

avanilde.kemczinski@udesc.br 
